\title{
Variations
}

Variations

Revue internationale de théorie critique

15 | 2011

La haine

\section{Anselm Jappe, Crédit à mort. La décomposition du capitalisme et ses critiques}

Clément Homs

\section{(2) OpenEdition}

1 Journals

Édition électronique

URL : http://journals.openedition.org/variations/107

DOI : 10.4000/variations. 107

ISSN : 1968-3960

Éditeur

Les amis de Variations

Édition imprimée

Date de publication : 1 mars 2011

Référence électronique

Clément Homs, "Anselm Jappe, Crédit à mort. La décomposition du capitalisme et ses critiques »,

Variations [En ligne], 15 | 2011, mis en ligne le 01 février 2012, consulté le 22 septembre 2020. URL:

http://journals.openedition.org/variations/107 ; DOI : https://doi.org/10.4000/variations.107

Les ami•e•s de Variations 


\section{Clément Homs}

Note de lecture

\section{Anselm Jappe, Crédit à mort. La décomposition du capitalisme et ses critiques (Lignes, 2011)}

Ce nouvel ouvrage d'Anselm Jappe est un second moment d'écriture qui suit logiquement le réassemblage de la critique de la valeur qu'il avait proposé au public francophone dans son ouvrage Les Aventures de la marchandise (2003). Composé de divers articles rédigés entre 2007 et 2010 que l'on peut très bien lire séparément, ce nouvel ouvrage s'applique à interpréter de manière illustrative et quasi méthodique certains des traits marquants de la seconde moitié de la première décennie de ce siècle (la fin de l'art, la marchandisation de la culture, la destruction de la planète, la fin de la politique, le recours à la violence, l'autodestruction du capitalisme...), au regard du fondement théorique posé et rassemblé précédemment. Et c'est le premier intérêt de l'ouvrage de ne pas être un simple commentaire inquiet sur « la crise », mais d'adopter une grille de lecture forte et originale, et qui plus est en rupture à la fois avec les appels à une moralisation du capitalisme comme avec l'interprétation «marxiste traditionnelle ». C'est donc seulement après cette longue traversée du désert théorique (qui commence pour cet auteur dès 1993 avec la parution de son premier essai théorique sur Guy Debord) rendue nécessaire par l'effondrement et l'inadéquation des grandes idéologies, qu'Anselm Jappe cherche désormais à appliquer dans cet ouvrage cette théorisation qualifiée de «critique de la valeur» et développée dans toute une mouvance multiforme qui va des revues allemandes Krisis et Exit! (dont le principal théoricien et inspirateur est Robert Kurz), à l'ouvrage fondateur de l'américain Moishe Postone, Temps, travail et 
domination sociale (Mille et une nuits, 2009) ${ }^{1}$. Pour autant, ce présent ouvrage n'a pas pour clé nécessaire la lecture du précédent car il contient à des moments opportuns des résumés de la critique de la valeur, si bien que cet ouvrage peut aussi se concevoir comme une sorte d'introduction à celle-ci.

Le livre étant sous-titré « la décomposition du capitalisme et ses critiques », ces dernières sont vivement prises à parti dans les divers articles afin de dégager ce qui paraît valable chez elles et ce qui leur échappe pour saisir la réalité de la crise contemporaine du capitalisme. Car pour l'auteur, plusieurs de ces critiques ont trop longtemps pensé que le caractère capitaliste de la production apparaissait extérieurement au procès de travail, travail qui en lui-même d'après ces théorisations devait être complètement naturalisé en tant que créateur « en soi » de toute richesse et comme simple activité instrumentale, comme si le travail était en quelque sorte l'essence générique de l'homme captée extérieurement par le capital. Comme l'a montré Postone, le capitalisme est ainsi défini par le « marxisme ricardien » (selon l'expression de Hans-Georg Backhaus²) comme un mode de distribution critiqué « du point de vue du travail ». Autrement dit, le marxisme traditionnel a considéré la notion de travail mobilisée par Marx comme la forme transhistorique du métabolisme avec la nature alors que l'analyse que le Marx de la maturité fait du travail concerne le travail sous le capitalisme. Ces visions du « marxisme traditionnel» ont alors mis seulement au centre de leur réflexion la théorie de l'exploitation du surtravail et celle de l'appropriation privée du surproduit social par la classe capitaliste, proposant pour seule critique du capitalisme, un changement du mode de distribution de la valeur qui se voit de par la naturalisation de son contenu - le travail -

1 Je me permets de signaler la très juste note de lecture de Dominique Méda sur cet ouvrage publiée dans la Revue française de socio-économie, $\mathrm{n}^{\circ} 6$, second semestre 2010, p. 175-182.

2 La critique de la valeur est en quelque sorte une poursuite des débats qui ont eu lieu à partir des années soixante au sujet de la découverte notamment au travers de ses racines hégéliennes, d'un Marx « hétérodoxe », chez des théoriciens comme Theodor Adorno, Herbert Marcuse, Roman Rosdolsky, HansGeorg Backhaus, Helmut Reichelt. Dans cette veine, en France, toute une filiation de théoriciens poursuivirent ces réflexions comme Jean-Marie Vincent ou André Gorz, avec à des moments une grande proximité avec des auteurs comme Robert Kurz ou Moishe Postone. 
complètement acceptée ${ }^{1}$. A propos des luttes contre les licenciements (comme pour les « récupérations » d'usine) ou des gens inquiets de perdre leur travail, Jappe estime ainsi, à rebours de la gauche, que leur colère «n'a rien d'émancipateur en tant que tel». C'est que pour les thuriféraires du capitalisme comme pour sa critique marxiste (critique qui est restée immanente aux formes sociales capitalistes), « la marchandise, l'argent, et la valeur sont des choses qui “ vont de soi " et qu'on trouve dans presque toutes les formes connues de vie sociale à partir de la Préhistoire. Les mettre en discussion semble aussi peu sensé que contester la force de gravitation. Une discussion n'est plus possible que pour ce qui regarde le capital et la plus-value, les investissements et les salaires, les prix et les classes, donc lorsqu'il s'agit de déterminer la distribution de ces catégories universelles qui règlent les échanges entre les hommes. C'est là le terrain où peuvent se manifester les différentes conceptions théoriques et sociales ${ }^{2} \gg$. A l'inverse de ces visions, la critique de la valeur et du fétichisme de la marchandise soutient que le capitalisme doit être saisi par une analyse plus profonde, en tant que forme particulière et inédite de vie et de socialisation, comme forme radicalement différente de médiation sociale constituée par le travail sous le capitalisme, un travail qui n'a rien d'une essence sociale transhistorique (d'où le Manifeste contre le travail du groupe Krisis auquel Jappe a appartenu ${ }^{3}$ ). L'auteur égratigne ainsi à plusieurs reprises les théoriciens les plus connus de cette gauche radicale, altermondialiste ou marxiste. Negri, Badiou, Zizek ou l'Insurrection qui vient en prennent chacun à leur tour pour leur grade et le chapitre consacré à la violence politique est une mise en garde sans détour à Julien Coupat et ses amis : «si la guerre civile - la vraie - éclatait, il ne serait pas difficile d'imaginer qui seraient les premiers à se trouver réveillés en pleine nuit et collés au mur sans façon, tandis qu'on viole les femmes et qu'on tire sur les enfants...» (p. 90).

\footnotetext{
${ }^{1}$ « Le socialisme note Postone, est vu comme un nouveau mode d'administration politique et de régulation économique du même mode de production que le capitalisme a engendré : il est pensé comme une forme de distribution non seulement plus juste, mais aussi plus adéquate à la production industrielle », in Temps, travail et domination sociale [noté dorénavant TTDS], Mille et une nuits, 2009, p. 24.

2 Anselm Jappe, Les Aventures de la valeur. Pour une nouvelle critique de la valeur, Denoël, 2003, p. 27.

3 Groupe Krisis, Manifeste contre le travail, UGE, 10/18, 2001 (1999).
} 
Quelle est alors la spécificité de cette saisie des bases sociales de la production capitaliste dont Anselm Jappe se réclame ? Prenons seulement un angle précis. Dans la société déterminée par la marchandise, c'est-à-dire sous le capitalisme, le travail quel qu'il soit, a toujours une double face avait-il montré dans son livre précédent. À première vue, le travail semble être seulement concret, et détermine la face d'usage d'une marchandise. Mais il possède aussi une autre face de par sa situation, une face abstraite, qui dérive de la fonction socialement médiatisante que possède le travail ${ }^{1}$. C'est là un caractère social historiquement spécifique du travail sous le capitalisme. Cette face abstraite de tout travail (qui est appelée le «travail abstrait») est comme un « objet fantomatique » (R. Kurz), c'est-à-dire invisible, mais constitue pour autant l'essence sociale de la société capitaliste-marchande car il y opère sa «synthèse sociale ». C'est cette face abstraite socialement médiatisante - du travail qui va être le contenu de la valeur et donc du capital (de la valeur qui se valorise au travers d'un processus social de dépense tautologique du travail). Ce n'est pas l'objectivation de la dépense de travail en soi qui constitue la valeur. La valeur est plutôt l'objectivation de cette fonction sociale particulière du travail sous le capitalisme. La substance sociale de la valeur, qui est donc aussi l'essence déterminante de la société capitaliste, est donc le travail dans sa fonction historiquement déterminée d'activité socialement médiatisante. La valeur est alors forme temporellement déterminée de richesse sociale et de médiation sociale objectivée, propre à la seule formation sociale capitaliste où les rapports sociaux sont constitués pour la première fois par le travail. C'est alors le travail - et non pas la séparation entre l'acte d'usage et

\footnotetext{
${ }^{1}$ « D'un côté note Postone, c'est un type de travail spécifique qui produit des biens particuliers pour d'autres ; mais d'un autre côté le travail, indépendamment de son contenu spécifique, sert au producteur de moyen pour acquérir les produits des autres. En d'autres termes, le travail devient un moyen particulier pour acquérir des biens dans une société déterminée par la marchandise ; la spécificité du travail est abstraite des produits qu'on acquiert par le travail (in TTDS, p. 223). Dans les sociétés où ne prédominent pas la production et l'échange marchands et où la distribution sociale du travail et de ses produits s'effectue par le biais d'un large éventail de coutumes, de liens traditionnels, de rapports de pouvoir, le travail est distribué par des rapports sociaux manifestes mais dans la société caractérisée par la forme marchandise, alors c'est le travail lui-même qui remplace ces rapports en servant de moyen objectif par lequel on acquiert les produits des autres. C'est-à-dire, précise Postone, qu'une nouvelle forme d'interdépendance vient de naître : personne ne consomme ce qu'il produit mais le travail ou le produit de chacun fonctionne comme moyen nécessaire pour obtenir les produits des autres » (in TTDS, p. 224). Telle est la spécificité du travail sous le régime capitaliste : il est l'activité socialement médiatisante.
} 
l'acte d'échange comme le pensait encore Alfred Sohn-Rethel ${ }^{1}$ - qui rend possible qu'une abstraction réelle conduise la société. Ici la valeur n'est plus une catégorie transhistorique comme dans sa compréhension par le « marxisme ricardien ». Avant même de critiquer la distribution de la valeur et la formation de la survaleur, une critique anticapitaliste doit saisir selon lui que derrière la valeur se cache déjà un rapport social de production particulier, que l'on doit comprendre comme un lien social objectivé, une forme de vie sociale historiquement inédite car propre à l'interdépendance sociale sous le capitalisme constituée par le travail. Critiquer ainsi le capitalisme au niveau de ses structures profondes, c'est donc d'abord critiquer cette forme sociale, la valeur.

C'est presque avec prudence, sans programmatisme ou promesses de lendemains qui chantent forcément, que l'auteur va aborder de manière éparse dans les articles, les « pistes » probables à emprunter. L'émancipation sociale remarque-t-il, si elle n'a rien d'automatique, reste possible car la reproduction humaine ne s'est pas toujours basée sur le mouvement de la valeur marchande. Cela existe peut-être sous sa forme complète, depuis quelque chose comme deux cents ans, et cela n'a pris en profondeur toute la structuration profonde de la société que depuis la Deuxième guerre mondiale. C'est donc certes un phénomène historique très important mais qui n'est certainement pas destiné à durer pour toujours. On peut très bien penser poursuit-il, que la forme de vie sociale que nous connaissons ne soit finalement qu'une « parenthèse dans l'histoire de l'humanité ». Que faire alors ? Parce que la critique de la valeur veut aller au-delà de la conception du rapport social capitaliste en termes de rapport d'appropriation juridique-externe de la survaleur par un groupe particulier et parce que le double caractère de cette forme de vie sociale et sphère séparée de la vie qu'est le travail et non le marché et la propriété privée des moyens de production, constitue le noyau social du capitalisme, cette critique met la barre de l'émancipation sociale à un niveau qui se veut plus profond et qui empêche complètement de voir dans la «politique » une

\footnotetext{
Voir la critique que fait A. Jappe à A. Sohn-Rethel, dans sa préface au recueil d'articles de ce dernier, $L a$ pensée-marchandise, Le croquant, 2010.
} 
solution. Car il ne s'agit plus de s'approprier les moyens de production pour les faire marcher à l'aune de l'intérêt général de la société c'est-à-dire de libérer le travail du capital, il s'agit de se libérer du travail en tant que tel. Et cela non pas en faisant travailler les machines à la place des humains car le mode industriel de production est la «matérialisation du procès de valorisation » remarquait déjà Postone ${ }^{1}$ (la technologie n'est pas neutre comme l'a cru le marxisme traditionnel toujours favorable au productivisme et à l'industrialisation), mais en abolissant une activité posée au centre de la structuration profonde d'une vie sociale qui n'a pas toujours été comme telle. « La seule chance, écrit Jappe, est celle de sortir du capitalisme industriel et de ses fondements, c'est-àdire de la marchandise et de son fétichisme, de la valeur, de l'argent, du marché, de l'Etat, de la concurrence, de la Nation, du patriarcat, du travail et du narcissisme, au lieu de les aménager, de s'en emparer, de les améliorer ou de s'en servir » (p. 52). L'émancipation sociale se situe ainsi dans le basculement des médiations sociales structurantes de «notre » société capitaliste-marchande (le travail, l'argent, la valeur, le capital...) vers d'autres formes de médiations moins fétichistes et moins mutilantes, et cela ne peut se faire que dans le cadre de la grande échelle sociale. Il faut qu'une société d'individus librement associés ne se médiatise plus à travers la production de marchandises et la valorisation de la valeur. Il faut inventer d'autres médiations sociales, il faut trouver un type de « synthèse sociale » autre que celui opéré sous le capitalisme, il faut changer les structures profondes de l'être social sous le capitalisme. Mais se demandant pourquoi il est si difficile d'imaginer réellement la fin du capitalisme, il note : « L'idée même suscite une peur bleue. Tout le monde pense avoir trop peu d'argent, mais chacun se sent menacé dans son existence, même sur le plan psychique, si l'argent fait mine de se dévaloriser et de perdre son rôle dans la vie sociale. [...] Il y a un accord général au moins sur une chose : il faut continuer à vendre, à se vendre, et à acheter. [...] Il faudrait combattre le sujet automate du capital, qui habite également dans chacun de

\footnotetext{
M. Postone, in TTDS, op. cit., p. 478. Jappe remarquait aussi qu' « une critique du capitalisme sans critique de la société industrielle est aussi insensée qu'une critique de la société industrielle sans critique du capitalisme », rapporté dans le texte de Jaime Semprun, " Notes sur le Manifeste contre le travail du groupe Krisis » dans un livre écrit avec René Riesel, Catastrophisme, administration du désastre et soumission durable, Editions de l'Encyclopédie des nuisances, 2008, p. 130.
} 
nous, et donc une partie de nos habitudes, goûts, paresses, inclinations, narcissismes, vanités, égoïsmes... Personne ne veut regarder le monstre en face. » Mais si personne ne veut penser à la fin du capitalisme, la crise profonde de la forme dominante de la socialisation, peut mettre d'après l'auteur le problème au calendrier historique. C'est là toute une autre dimension de l'ouvrage et de sa théorisation.

Car les marxistes remarque-t-il, quelles que soient leurs différences par ailleurs, ont toujours lu et lisent encore la théorie de la crise chez Marx comme une théorie de la distribution inégale de la richesse (distribution inégale qui trouverait aujourd'hui sa source dans la spéculation, la dérégulation, la recherche de surprofits sur les marchés financiers). Pour Jappe au contraire, c'est là méconnaître l'existence même d'une «limite interne» au capitalisme ${ }^{1}$. Parce qu'une crise d'autodestruction du capitalisme serait inscrite « in nuce » dans sa logique de base, une théorie du capitalisme est forcément une théorie de sa crise permanente et absolue (une « théorie de la crise » reformulée notamment par Robert $\mathrm{Kurz}^{2}$ ). C'est là un des intérêts majeurs du livre, l'exposition d'une nouvelle structure théorique qui permette de comprendre un processus de crise du capitalisme qui a commencé dans les années 1970. Quel est le gros de son propos ? Cette dynamique folle et autodestructrice du capitalisme dérive du fait que plus il développe sa logique, plus le capital perd sa substance (le travail abstrait) et donc plus la valeur s'effondre, car telle serait la contradiction nécessaire de sa logique de base : «devoir réduire - à cause de la concurrence - par des technologies et de la rationalisation, le travail humain qui constitue en même temps et

Remarquons qu'André Gorz parle abondamment de cette « limite interne » du capitalisme, que peu de critiques contemporaines veulent reconnaître, dans son livre Ecologica, Galilée, 2008.

2 Robert Kurz, et Moishe Postone développent deux critiques de la valeur qui divergent sur un point central. Pour Kurz, avec les gains de productivité, le capital perd sa substance (le travail abstrait) et, avec la troisième révolution industrielle, la microélectronique, il la perd absolument. Pour Postone, au contraire, les gains de productivité accroissent la valeur, mais provisoirement. Dès lors que le gain de productivité s'est généralisé, l'accroissement de valeur s'annule, l'unité de base du travail abstrait (l'heure de travail) étant ramenée à son niveau initial. Ainsi, pour Kurz la valeur s'effondre (il y a une limite interne absolue), alors que pour Postone, la valeur s'accroît sans cesse puis revient à son point de départ. Pour une critique de Postone sur ce point, voir R. Kurz, " La substance du capital », revue Exit, $2004,{ }^{\circ} 1$ (on retrouve ce texte en allemand sur le site internet de la revue). 
paradoxalement la seule source de la valeur » (p. 195). Par l'augmentation du capital fixe qui permet des gains de productivité, la substance sociale du capital (le travail abstrait) est toujours plus réduite et tend avec les gains de productivité issus de la « révolution de la microélectronique » vers une limite absolue où le capital n'est plus capable de reproduire la société qu'il constitue de par son mouvement. Se faire exploiter devient alors un privilège rare. Dans les centres capitalistes occidentaux où l'appareil productif est de plus en plus automatisé, les humains sont ainsi de plus en plus « non-rentables » et donc « superflus » pour la machine sociale de la valorisation et la société fétichiste capitaliste. Dans les centres capitalistes (les pays de la Triade), les masses de travailleurs improductifs de valorisation croissent sans cesse. Plus de $25 \%$ de l'activité mondiale est ainsi assurée par deux cents multinationales qui emploient seulement $0,75 \%$ de la population mondiale, c'est-à-dire que la population directement productive de capital (travail productif) représenterait probablement d'après André Gorz, moins de 10\% de la population active des pays dits développés ${ }^{1}$. Ainsi, au fur et à mesure d'un certain niveau d'incubation de sa propre logique mortelle, le capitalisme est ainsi un système fou et aveugle qui s'effondre sur lui-même car il scie la branche sur laquelle il est pourtant assis. Mais attention remarque Jappe, cette crise profonde ne débouche sur aucun dépassement positif et automatique du capitalisme, l'espace ouvert n'est que celui d'un vaste « processus de barbarisation » qui n'a rien des lendemains qui chantent. Au travers des politiques néolibérales dégraissant l'Etat social bourgeois et du virage du «capitalisme vert», partout s'installe une administration du désastre capitaliste qui évacue de la reproduction humaine les « déchets » humains (Zygmunt Baumaun).

Loin de comprendre la crise de 2008 (comme l'éclatement des bulles précédentes) comme une offensive du capital (la crise comme stratégie du choc) et de penser que le capitalisme est finalement en bonne santé, ce à quoi nous assistons est au contraire une crise mortelle pour le capitalisme. Le néolibéralisme, la mondialisation, la financiarisation ne sont pas des signes de 
bonne santé et d'offensive du capital soutient l'auteur, mais plutôt des expressions visibles de sa fuite en avant vers sa « limite interne ». Tous ces phénomènes n’ont formé que des « béquilles » $(\mathrm{p}$. 110) à la crise profonde de la valeur. Finalement écrit Jappe, « le néolibéralisme était, au contraire, la seule manière possible de prolonger le système capitaliste » (p. 109). L'échelle nationale de la production de valeur comme les « faux frais » qui constituaient l'Etat social Providence (permis par une ponction sur la valorisation capitaliste assise durant les Trente Glorieuses sur des gains de productivité importants), devaient forcément être transformés et réduits, au vu de l'effondrement de la valeur. A l'inverse de la critique anti-libérale, Jappe se refuse à donner toute la faute de la crise aux spéculateurs, à l'oligarchie (thème très à la mode), aux mégabanques ou aux politiciens corrompus et faire une chasse à un groupe limité de personnes pour tenter de sauver le système, comme le font les populismes de droite comme de gauche. La crise n'est que superficiellement une « crise financière », c'est une crise de l' « économie réelle », une crise du capitalisme. La croissance des années 1980 et 1990 ne fonctionnait déjà plus sur aucune base autonome réelle, la société capitaliste n'a ici réussi à prolonger son existence qu'en consommant sa croissance future espérée au travers du crédit. La nouvelle économie des bulles spéculatives qui naissait, n'était pas un dépassement de la constitution de la synthèse sociale par le travail créateur de valeur, mais une autre béquille provisoire pour masquer que le roi était nu, les bulles de ces dernières décennies étant de plus en plus rapprochées, importantes et leurs conséquences toujours plus dévastatrices. Si «le crédit, qui est un profit consommé avant d'avoir été réalisé, peut reporter le moment où le capitalisme atteindra ses limites systémiques, mais il ne peut pas l'abolir. Même le plus bel acharnement thérapeutique doit se terminer un jour » (p. 111). La bulle spéculative va ainsi crever un jour, on découvrira que depuis des décennies finalement le système capitaliste mais aussi tous les Etats et les personnes qui vivent qu'au travers du mouvement de la société capitaliste-marchande, ne vivent qu'à crédit. La société capitaliste ne simulant plus sa «bonne marche » qu'au travers du crédit, il y a toujours une tendance à continuer à engager des sommes toujours plus grandes, peut- 
être jusqu'à la mort.

Toute une autre partie du livre développe aussi à partir de la «théorie de la crise », une compréhension intéressante de la signification profonde de la crise écologique généralisée. Car pour Jappe, la logique de la valeur conduit non seulement à une « limite interne » absolue, mais aussi à une «limite externe» située dans la nature. Face au mécanisme de l'effondrement de la valeur incorporée par marchandise (des produits de moins en moins chers pour être compétitifs) qui pousse aussi à la baisse de la survaleur obtenue par marchandise, il existe un seul phénomène (provisoire) de compensation pour ces entreprises plongées dans la guerre économique intercapitaliste de la concurrence. Si la survaleur obtenue par marchandise est moindre, il faut compenser cela par l'augmentation de l'échelle de la production de marchandises, il faut produire toujours plus en utilisant davantage de matières premières pour fabriquer toujours plus de marchandises afin de tenter d'annuler l'effondrement de la valeur. Mais si la valeur n'augmente plus guère dans les centres capitalistes depuis trente ans, en revanche, pour rester sur ce « sur place », ce qui augmente et accélère c'est la consommation de ressources, la pollution et la destruction. La société capitalistemarchandise est la première société dont le dynamisme aveugle est autodestructeur, car «le capitalisme, écrit Anselm Jappe, est comme un sorcier forcé à jeter tout le monde concret dans le chaudron de la valorisation et la marchandisation, pour éviter que tout s'arrête ${ }^{1} \gg$. Le capitalisme évite ainsi sa fin (la réduction de la valeur) en courant toujours plus vite que la tendance qu'a la valeur à s'écrouler sous son propre poids, et grâce à une augmentation continuelle de la production matérielle ravageant tout le monde concret. Les ruines du monde concret dévastées sont le trognon de pomme qui reste après le passage de la logique de la valeur, car le concret n'est que le support du processus aveugle de valorisation. La prise en compte de la finitude du monde concret, comme le croient certains, est totalement impossible au sein d'un tel monde social déterminé par la logique abstraite de la valeur qui meut la totalité de cette société présente. Ce serait s'aveugler que de Anselm Jappe, in « Décroissants encore un effort ! », Crédit à mort, Lignes, 2011, p. 194. 
penser de manière extrémiste que l'on puisse réguler le capitalisme comme on l'entend à gauche et chez certains écologistes, ce serait s'aveugler parce qu'il régule déjà l'ensemble de la vie sociale moderne dans les «conditions sociales muettes» (Marx) qu'il constitue, parce que cette crise écologique généralisée est déjà inscrite dans le déploiement de sa logique de base qui est sa forme même d'existence. Sur ce point soyons en sûr, pour Jappe, la société capitaliste-marchande ne sera jamais éco-compatible. On ne chevauche pas le tigre lancé dans une course folle. La crise écologique généralisée ne peut pas trouver de solution dans le cadre de la société capitalistemarchande. Il faut sortir totalement de ce monde social là en faisant basculer l'ensemble des médiations sociales présentes vers une autre forme radicalement différente de vie sociale. Dans cette interprétation proposée par l'auteur, la finitude de la planète si elle est un fait naturel, c'est son agression par la dynamique de la forme capitaliste de vie sociale qui est sociale et historique. Cette forme historiquement spécifique d'agression est la matérialisation concrète et visible de la forme dynamique, aveugle, folle et autodestructrice spécifique à la société capitaliste-marchande qui se crée et s'avance devant elle-même, comme un mur infranchissable, sa propre limite externe. Et plus le capitalisme s'approche de sa limite interne absolue, plus il se rapproche de manière concomitante de sa limite externe. Cette forme d'agression est l'envers, c'est-à-dire l'autre face nécessaire de la forme de dynamique autodestructrice de cette société. Cette forme d'agression vis-à-vis de la planète ne peut être naturalisée ou être comprise de manière transhistorique comme le fait de l'action instrumentale et générale de l'homme sur la nature (par exemple en partant de la « révolution néolithique » pour expliquer la forme d'agression contemporaine sur la nature, comme chez Michel Bounan ou Philippe Godard). Certainement que d'autres formes de société composées d'autres rapports sociaux ont aussi exercé une forme d'agression sur la nature sur l'île de Pâques ou ailleurs, mais la forme d'agression à laquelle présentement nous avons à faire, doit être saisie en rapport avec la société spécifique contemporaine qui l'a engendré. La crise écologique généralisée doit être comprise de manière historiquement spécifique, c'est-à-dire comme étant le déploiement 
logique de la dynamique nécessaire mais autodestructrice de la société mue par la valeur, et non comme une sorte de « démesure » anthropologique et morale liée à une prétendue nature humaine qui reste tout particulièrement une illusion occidentale ${ }^{1}$. L'écologisme radical doit certainement mieux spécifier sa critique au lieu de démarrer sur des grands discours universels et généraux sur la « nature humaine », l'« hybris », « l'humanité », voire parfois « le cours de toute l'histoire humaine depuis le néolithique », qui ne débouchent souvent que sur un appel à plus de morale voire de sacré quand ce n'est pas une volonté de participer à l'administration du désastre en organisant le rationnement collectiviste dans le cadre d'une « décroissance économique » improbable ${ }^{2}$.

Dans un texte intitulé «Politique sans politique », la question de la compréhension de la forme politique sous le capitalisme est aussi largement développée par l'auteur. Pour beaucoup de groupes et partis de la gauche électorale (même radicale), la politique est la sphère sociale qui permettrait d'imposer des « limites au marché » (réguler et moraliser le capitalisme), ou encore pour cette gauche la «démocratie» serait l'espace politique de la «libre décision consciente et collective ». Comme le croient les simples antilibéraux et la gauche en général qui n’ont pour seule proposition qu'une immense nostalgie pour le capitalisme productiviste à papa de l'Etat social Providence des Trente Glorieuses (contre lequel il y a eu pourtant un « 68 mondial »), le capitalisme serait par nature contre toute instance supérieure régulant l'intérêt général. Nombreux parmi eux dénoncent aujourd'hui une forme spectaculaire de la politique pour mieux affirmer un « retour » à ce que serait la «vraie politique ». Là encore l'auteur prend à rebrousse poil tous ces discours convenus qui ne veulent pas aller au fond des choses et voir la totalité sociale que constitue la société capitaliste-marchande. Jappe parle ainsi d'une confusion autour du concept de «politique » : certes remarque-t-il, «si on l'identifie avec l'agir collectif, avec l'intervention consciente des hommes dans la société, avec un " amour du monde " (Arendt), il est évident que personne ne

Marshall Sahlins, La nature humaine, une illusion occidentale, L'éclat, 2010.

2 Voir à ce propos le livre de Jaime Semprum et René Riesel, Catastrophisme, administration du désastre et soumission durable, op. cit. 
saurait être contre, et une "critique de la politique" ne pourrait se concevoir que comme une simple indifférence au monde» (p. 56). Or justement, la forme de l'agir collectif sous le capitalisme, que nous appelons de manière restreinte la «politique », n'a rien d'une forme transhistorique et naturelle. La philosophie politique (que Franck Fischbach a très bien critiquée dans son Manifeste pour une philosophie sociale, L'harmattan, 2009) a toujours réfléchi en termes très généraux et abstraits, de manière transhistorique et transculturelle. Pour l'auteur, il faut comprendre ce que relève la forme de l'agir collectif contemporain, comme quelque chose de structuré et d'immanent aux formes sociales capitalistes, la sphère politique sous le capitalisme [n'est pas extérieure et alternative à celle-ci. La sphère de l'État/politique serait née (à partir des formes politiques et étatiques précédentes) du fait qu'une société déterminée par l'échange généralisé de marchandises ne prévoit pas de relations sociales directes du fait de la nature de l'être social sous le capitalisme, par conséquent il faut une sphère particulière pour les rapports directs et pour la réalisation des intérêts universels, à moins de plonger le système dans la barbarie et la guerre civile des intérêts particuliers. C'est pourquoi la formation sociale capitaliste doit produire en elle, une instance séparée qui s’occupe de l'aspect général. La société capitaliste moderne «a besoin d'une instance qui se charge des structures publiques sans lesquelles elle ne pourrait pas exister. Cette instance est l'Etat, et la " politique " au sens moderne (et restreint) est la lutte menée autour de son contrôle » (p. 58). La « politique » et «l'économie » ne sont alors que des sphères d'une même totalité sociale, des subsystèmes complémentaires entre eux. L'État moderne, dans sa forme contemporaine, est donc créé par la logique de la marchandise, la « politique » telle que nous la connaissons est une forme historiquement spécifique au capitalisme, une forme spécifique de l'agir collectif et sa dénaturation sous une forme déjà fétichisée. Conséquence de cette nature de la « politique », celle-ci n'a pas de moyen autonome d'intervention. L'agir collectif sous le capitalisme ne peut s'exercer qu'au travers du mouvement de la valeur. « La preuve visible : rien n'est possible en politique qui ne soit d'abord « financé » par la production marchande » (p. 58). Il devient alors 
évident que dans la société de la valeur la politique se trouve dans un rapport de dépendance vis-àvis de l'économie et de la croissance capitaliste. Lorsque l'État cherche à créer son propre argent en imprimant du papier-monnaie, cet argent se dévalorise très vite. Le pouvoir étatique fonctionne finalement seulement s'il réussit à opérer une saignée d'argent (l'impôt) sur des procès de valorisation réussis. Lorsque ces procès commencent à ralentir (crise de la valeur), l'économie limite et étouffe toujours plus l'espace d'action de cette forme de «politique » immanente à la société capitaliste : c'est alors l'apparition du néolibéralisme comme seule politique possible dans le cadre fou de la logique de la valorisation en crise. Néolibéralisme qui n'est pas le complot de tel ou tel groupe limité ou une simple idéologie conservatrice, mais une contrainte systémique que la société capitaliste s'inflige à elle-même comme béquille improbable à l'effondrement de la valeur. Avec la disparition de ses moyens financiers, l'État se réduit alors à la gestion, toujours plus répressive, de la pauvreté... Dans le cadre d'une telle interprétation, la politique « ne représente pas une instance « neutre », ni une conquête que les mouvements d'opposition auraient arrachée à la bourgeoisie capitaliste. En effet, celle-ci n'est pas nécessairement hostile à l'Etat ou à la sphère publique - cela dépend de la phase historique » (p. 58). C'est ainsi le capitalisme lui-même qui a très massivement recouru à l'État et à la politique pendant la phase de son installation (entre le XVe siècle et la fin du XVIIIe siècle) et qui a continué à le faire là où les formes sociales de bases du capitalisme devaient encore être introduites de force - dans les pays « arriérés » à l'est et au sud du monde au cours du XXe siècle dans les pays communistes et dans le Tiers monde, pour faire ce que Robert Kurz appelle la « seconde modernisation de rattrapage ». C'est seulement remarque Jappe, dans les périodes où le marché semble tenir sur ses propres jambes, que le capital voudrait réduire les « faux frais » qu'implique un État fort. Finalement au lieu d'être un point d'appui pour s'opposer au capitalisme, «cette forme de “ politique " est un mécanisme de régulation secondaire à l'intérieur du système fétichiste et non-conscient de la marchandise » (p. 58). «Dans l'arène politique remarque Jappe, on se dispute sur la distribution des fruits du système marchand - le 
mouvement ouvrier a joué essentiellement ce rôle $-[\ldots]$ mais non sur son existence elle-même » (p.58). Le personnel politique de la " gauche de gauche » comme les syndicalistes du mouvement ouvrier proposent seulement de défendre les intérêts sous forme déjà marchande des catégories sociales constituées par la logique fétichiste elle-même, du genre «pouvoir d'achat», mieux partager et redistribuer le gâteau de la forme capitaliste de richesse, dénoncer le « Contrat première embauche » $(\mathrm{CPE})$, pour mieux défendre le statut du contrat à durée indéterminée $(\mathrm{CDI})$, lutter contre les licenciements, taxer simplement les riches, etc.

Pour l'auteur il faudrait donc reformuler une théorie de l'Etat sous le capitalisme. On ne pourrait pas adopter une théorie transhistorique de 1'Etat, il faudrait aussi dégager une théorie de la forme étatique historiquement spécifique à une formation sociale capitaliste. Car on ne devrait pas confondre l'agir collectif au sens abstrait et transhistorique de la philosophie politique, avec la nature de l'agir collectif sous le capitalisme, agir qui serait complètement immanent aux formes sociales capitalistes. De ce fait, une critique du capitalisme devrait être aussi une critique de l'Etat et de la politique. Pour sortir du capitalisme, ils ne sont pas la solution. Cette antipolitique ou plutôt cette « politique sans la politique » comme dit l'auteur, refuserait la trahison de l'intention originale de l'« agir collectif » portée par les tenants contemporains de la « politique ». « Au lieu d'identifier la politique avec les institutions publiques de la société marchande, on peut identifier la politique avec la praxis en général. Mais cette praxis, il ne faut pas l'opposer abstraitement à la théorie. La théorie dont il est question ici n'est pas la servante de la praxis, ni sa préparation, mais en est une partie intégrale » (p.66). Et il propose et discute les «formes futures de praxis » que pourrait prendre cette autre forme de politique qui cherche la séparation radicale d'avec le monde de la « politique » et de ses institutions, de la représentation et de la délégation, pour inventer à leur place de nouvelles formes d'intervention directe. 
Mais touchant aussi à des réflexions sur la culture, l'art, l'utopie, la subjectivité narcissique contemporaine, le paradigme maussien du don ou encore le concept de «common decency» de Jean-Claude Michéa, l'ouvrage apporte encore bien des réflexions stimulantes sur le monde contemporain.

Clément Homs 